\title{
Are Perceived Self-Management Skills protective against psychological morbidity? A study with metastatic breast cancer patients.
}

\section{Luzia Travado ${ }^{1}$, Joaquim C Reis ${ }^{2}$, Michael H Antoni ${ }^{3}$, Berta Sousa ${ }^{4}$}

${ }^{1}$ Champalimaud Clinical and Research Center, Champalimaud Foundation, Psycho-oncology program-Neuropsychiatry Unit, Lisbon, Portugal. 2Instituto de Biofísica e Engenharia Biomédica, Faculdade de Ciências, Universidade de Lisboa, Lisbon, Portugal

${ }^{3}$ Sylvester Comprehensive Cancer Center- University of Miami Miller School of Medicine, Department of Psychology-and Cancer Control Program, Miami, USA.

4 Breast Unit, Champalimaud Clinical Center, Champalimaud Foundation, Lisbon, Portugal

\section{BACKGROUND}

Previous studies indicated that higher levels of Perceived SelfManagement Skills (PSMS) are associated with lower levels of emotional distress in non-mBCa patients. However, there are no such studies with metastatic breast cancer (mBCa) patients ${ }^{1}$.

\section{OBJECTIVES}

The present investigation* aims to examine whether perceived self-management skills are associated with distress and psychological morbidity in metastatic breast cancer patients.

\section{SUBJECTS AND METHODS}

A total of 61 women with $\mathrm{mBCa}(59.9 \pm 10.6 \mathrm{yrs}$ old, range:39-84) who met the entry criteria, signed the informed consent, were submitted to ${ }^{18} \mathrm{~F}-\mathrm{FDG}$ PET/CT and completed psychosocial assessments related with negative affect (NA).

Eligible participants of this study were adult women ( $>18$ years of age) with metastatic or locally advanced breast cancer not amenable to curative treatment by surgery or radiotherapy, under treatment at the Breast Unit of the Champalimaud Clinical Center, in Lisbon, Portugal, for which a whole body FDG-PET study including brain data acquisition was prescribed as part of their work-up and best clinical practice. Only patients on first or second-line of treatment were recruited. Patients must have: ECOG Performance status 0 or 1 ; adequate bone marrow, coagulation, liver and renal function. Exclusion criteria include: history of brain or other CNS metastases, radiation therapy to the brain or skull lesions, history of neurodegenerative neurological diseases and current treatment with corticosteroids or IV chemotherapy.

An informed consent form was provided for each patient. Recruitment was conducted between June 2017 and May 2019. The study was approved by the local Institutional Ethical Committee.

Table 1: Prevalence of Distress/Negative Affect in $\mathrm{mBCa}$ patients.

\begin{tabular}{|c|c|c|}
\hline \multicolumn{2}{|c|}{ Cut-off scores of questionnaires* } & Prevalence of NA above \\
\hline BSI - global NA & $1,7^{* *}$ & 37,70 \\
\hline HADS-depression & 8 & 32,80 \\
\hline HADS-anxiety scale & 8 & 42,60 \\
\hline
\end{tabular}

${ }^{*}$ Clinical cut-off score indicates a score at which a subject has a greater probability of belonging to a clinical sample rather than a non-clinical sample ${ }_{* \star}$ BSI cut-off score for Portuguese population.

\section{Distress or Negative Affect measures}

Distress was measured by the Global Stress Index using the Brief Symptom Inventory (BSI) ${ }^{2}$

Depressive and Anxiety symptoms was measured using the Hospital Anxiety and Depression Scale (HADS) ${ }^{3}$

Perceived self-management skills measure

Perceived self-management skills was measured using the Measure of Current Status questionnaire (Carver, 2005) ${ }^{4}$. The MOCS subscale A measures participants' current self-perceived status on stress management skills, namely the ability to relax, recognize stress-inducing situations, restructure maladaptive thoughts, be assertive about needs, express anger appropriately, and choose appropriate coping.

\section{Statistical analysis}

A linear regression model was utilized: Global Stress Index as criterion (dependent) variable and age and educational level as covariates and MOCS as dependent variable.

\section{RESULTS}

The regression model was significant:

$F(3,41)=12,266, P<0,01, R^{2}=0,473$

Greater perceived self-management skills (PSMS) are predictive of distress in this sample of $\mathrm{mBCa}$ patients:

$$
\text { standardized } \beta=-0,665, t=-5,494, p<0,01
$$

Age and educational level did not contribute significantly to distress levels.

\section{CONCLUSION}

Higher levels of perceived self-management skills are significantly associated with lower levels of distress in this sample of $\mathrm{mBCa}$ patients.

These findings suggest the relevance of implementing stressmanagement interventions with $\mathrm{mBC}$ a patients to improve their self-management skills and coping with the disease to reduce their emotional distress. ${ }^{5}$

\section{REFERENCES}

1. Lattie EG, Antoni MH, Fletcher MA et al., (2012). Stress management skills, neuroimmune processes and fatigue levels in persons with chronic fatigue syndrome. Brain Behav Immun. ;26(6):849-58

2. Derogatis LR, Fitzpatrick M. The SCL-90-R, the Brief Symptom Inventory (BSI), and the BSI18. In: Maruish M, ed. The Use of Psychological Testing for Treatment Planning and Outcomes Assessment: Volume 3: Instruments for Adults. ; 2004

3. Zigmond AS, Snaith RP. The Hospital Anxiety and Depression Scale. Acta Psychiatrica Scandinavica. 1983;67(6). doi:10.1111/j.1600-0447.1983.tb09716.

4. Carver, C. Measure of Current Status. 2006. Retrieved January 17, 2014, from Department of Psychology, University of Miami Web site:http://www.psy.miami.edu/faculty/ccarver/

5. Reis JC, Antoni MH, Travado L. Emotional distress, brain functioning, and biobehavioral processes in cancer patients: a neuroimaging review and future directions. CNS Spectrums. process

Funded by FCT: DISTRESSBRAIN PROJECT, PTDC/MHC-PSC/3897/2014) (2016-2020) 